\title{
Oligella ureolytica conjunctivitis in a female patient
}

\author{
Giuseppe Ivan Potente, ${ }^{1}$ Francesca Fabiola Bianco, ${ }^{2}$ Raffaella Aiello ${ }^{3}$ \\ ${ }^{1}$ Laboratory of Chemical and Clinical Analysis and Microbiology, Department of Medicine, \\ ${ }^{2}$ Department of Biology, ${ }^{3}$ Department of Medical Biotechnology, Soveria Mannelli Hospital, \\ Catanzaro, Italy
}

\section{Summary}

Oligella ureolytica, an aerobic gram-negative found as a commensal microorganism in human urinary tracts, is an emerging bacteria which is rarely implicated as human pathogen. In the past referred to as CDC Group IVe, this bacterium is not commonly encountered as a source of infection and is difficult to isolate by means of conventional laboratory procedures. We present here the case of a 55-year-old female patient suffering from bilateral conjunctivitis. An Oligella ureolytica infection was identify in an eye swab sample. The pathogen was isolated in pure culture (48/72 h) and an antibiogram was determined by Kirby-Bauer disc diffusion method (manual test).

\section{Introduction}

Oligella ureolytica, which belongs to Moraxellacae's genus $(1,2)$, is a low-virulent and pathogenic organism that is now part of pseudomonaceae family. Oligella family is divided into Oligella urethralis and Oligella ureolytica.

O. ureolytica, known in the past as CDC group IVe, is an aerobic

Correspondence: Giuseppe Ivan Potente, Struttura Complessa Laboratorio Analisi Chimico-Cliniche e Microbiologiche, Ospedale di Soveria Mannelli, Viale Rubbettino, 88049 Soveria Mannelli (CZ), Italy.

Tel.: +39.320 .7680126 .

E-mail: ivanpotente65@gmail.com.

Key words: Oligella ureolytica; infectious diseases; conjunctivitis.

Contributions: the authors contributed equally.

Conflict of interest: the authors declare no potential conflict of interest.

Received for publication: 5 February 2016.

Revision received: 8 April 2016.

Accepted for publication: 8 April 2016.

(c) Copyright G.I. Potente et al., 2016

Licensee PAGEPress, Italy

Microbiologia Medica 2016; 31:5797

doi:10.4081/mm.2016.5797

This article is distributed under the terms of the Creative Commons Attribution Noncommercial License (by-nc 4.0) which permits any noncommercial use, distribution, and reproduction in any medium, provided the original author(s) and source are credited. gram-negative motile bacterium with peritrichi and flagella, generally isolated from cervical lymph nodes and human urine (1).

Oligella urethralis is a less common species: it is non-motile, urease-negative, it does not convert nitrate to nitrite and is generally susceptible to most antibiotics including penicillin (2).

Our Oligella ureolytica's records can be correlated in the literature cited in Pubmed and other scientific major links.

We present here the first case of bilateral conjunctivitis in Italy, in a 55-year-old female patient, caused by Oligella ureolityca.

\section{Case Report}

A 55-year-old female patient, mussels' sales clerk and therefore used to cleanse the mollusks, in apparent good health and without any predisposition to eye diseases, asked us for treatment to our laboratory due to a bilateral conjunctivitis.

She suffered from an intense secretory burning, more accentuated in the morning, and photophobia with a sense of general illness. She had those symptoms from about one week and an ophthalmologist sent her to our laboratory.

Through an ocular swab, a secretion sample from both her ocular conjunctiva (right and left) was collected.

We used a STUART swab, a ready-to-use sterile system, supposed to be collect, transport and preserve clinical specimens for bacteriological examination.

Stuart Transport Medium is a non-nutritional medium that maintains the viability of organisms without significant multiplications. The small agar content provides a semi solid consistency, which prevents oxidation and drying during transport. Sodium thioglycolate was added to produce a reduced environment to improve the recovery of anaerobes. Methylene blue is the oxidation-reduction indicator (4).

Both samples were propagated by a sterile swab on four different agar plates, two different Blood agar (CNA and COL), MacConkey, Sabouraud, that were dissolved by using a sterile loop and all the plates were, later, put in a thermostat in $5-10 \%$ of $\mathrm{CO}_{2}$ and a temperature of $35-37 \mathrm{C}^{\circ}$.

After 24 hours of incubation, no living colonies were observed. We thus left our samples growing and we were able, after 48 hour, to see a modest amount of colonies in the two blood agar plates (CNA and COL).

The colonies were small, white, opaque with a continuous margins, and non-haemolytic.

Vitek2 compact system cards (BioMèrieux, Mercy-L'etoile, France), specific for Gram-negative bacterial family (GN REF 21341), were used to analyse and identify the pathogens that were grown in our plates, while Kirby-Bauer (KB) was used to test antibiotic resistance/susceptibility.

Afterwards, we re-plated the colonies on blood agar and, after 48 hours, when growth occurred, we proceeded to new identifications and susceptibility tests with the same procedures that had confirmed ours previous data. 
The results helped the doctor in choosing which antibiotics were more suitable to treat this pathology.

A KB test, following EUCAST guidelines, was performed: that procedure utilizes small filter disks impregnated with a known concentration of antibiotics. The disks were placed on a Mueller-Hinton agar plate that was inoculated with the tested microorganism. After the incubation antibiotics diffused from the disk into the surrounding agar.

If susceptible to one of the antibiotics, the tested organism wouldn't be able to grow in the area immediately surrounding the disk, displaying a zone of inhibition instead.

The antibiotics used for the identification of this bacterium are listed in Table 1.

The inhibition halos were accurately measured by using a millimetre ruler. The antibiogram did not highlight resistance in vitro against the tested antibiotics, facilitating in this way a targeted therapeutic approach.

Following the identification of Oligella and the performing of the susceptibility tests, the patient was administered with chloramphenicol and rolitetracycline during seven days.

\section{Discussion and Conclusions}

Oligella infection has rarely been reported in literature. This may be due to misidentification of the organism or uncertainty of its pathogenicity.

The few cases of pathogenic infection with Oligella ureolytica, described in the literature, have occurred in patients ranging in age from newborn to 89 years and from the varied locations of India, Turkey, Canada, and the United States (5).

Some issues in the identification of the pathogen can be linked to limitations in commonly available laboratory procedures which make difficult the identification of this bacterium: for example the incubation period is very long (4 days), and not all laboratories incubate cultures for that long. Furthermore, it is generally believed agreed that Oligella bacteria can be misidentified as phenotypically similar organisms, such as Bordetella bronchiseptica and Achromobacter.

Therefore Oligella ureolytica has gone unrecognised or was incorrectly identified because of laboratory technicians' and clinicians' lack of familiarity with this bacterium (5).

This organism is most recovered from urethral specimens and from urinary tract, where it is a commensal organism (1).

Despite these considerations, we have shown that Oligella could also become a pathogen, as in this case of the patient who has, probably, contracted the bacterium during the wash mussels in sale outlets.

On a worldwide basis, one of the principal risks associated with the consumption of bivalve molluscs' could come from a microbiological contamination of waters in which they grow or from the failure of their purification procedures, especially when the mussels are meant to be eaten raw.

They often, present the constituting valves covered with algae and fouling, that have to be removed with a special treatment. This decontamination practice was performed by some machineries, constituted by a metal cylinder containing a drum and a rotating mechanism

Table 1. Antibiotics used for the identification of Oligella infections.

\begin{tabular}{lc} 
Antibiotics & Concentration, $1 \mathrm{~g}$ \\
\hline Amoxicillin & 30 \\
Chloramphenicol & 10 \\
\hline Chloramphenicol & 30 \\
Levofloxacin & 10 \\
\hline Ampicillin-Sulbactum & 10 \\
\hline
\end{tabular}

(which causes the scrambling of the shellfish and the collision/friction between them), in a bath of potable water that is connected to the system and that will be eliminated at the end of the operation, through the exhaust system. The shock and the friction of the valves whit one another, along with the contextual rinsing, determine the detachment and the removal of the unwanted material. The molluscs', so treated, are subjected to stress conditions, which make them ready to be eaten by the consumer. The purification removes only low or moderate levels of the microbial contamination; therefore, it cannot be used for treating bivalve molluscs, that are strongly contaminated (3).

Moreover, not all bacteria or viruses can be successfully removed from this purification process, thus becoming easily pathogenic for humans, as in this case.

Our female patient had a clear bacterial conjunctivitis with particularly debilitating symptoms, as evidenced by the ophthalmologist: in fact, she had a significant conjunctival hyperemia with contextual blepharitis and filled secretion. This situation induced a marked photophobia and a general discomfort with strong difficulty in performing even routine activities.

After the execution of the diagnostic swab and subsequent administration therapy, the patient was sent back to our attention. A new check swab was performed to both conjunctiva, which gave negative results with a set of symptoms in regression, in conjunctival hyperemia and in the palpable reduction of nuisance, related to the exposure to light. The patient recovered after receiving the treatment.

This particular employment of the patient could have played a role in the induction of those adverse events because of water contamination sourced by mussels. An important observation that must be made: the discontinuity in the use of protection devices could have played an important role in the contamination, considering that the patient was in good health and without medical history family in eye diseases.

Therefore it gets important to use personal protective equipment that, if used properly by workers, can certainly lead to reduction of the risk related to this cluster type of pathology.

The lack of other data, since the unique of the Oligella's human contamination, considering the healthy state of the patient and that her employment was the only way of contamination with the bacterium, means that ours are only assumptions.

Advanced laboratory techniques will lead us to more recognized cases and further studies are necessary to understand this bacterium's clinical significance.

In addition to it, it should be noted also that, even a basic laboratory, if it operates properly and carefully, in collaboration with the specialists that request the examinations, can obtain even the most difficult diagnosis of unknown and not identifiable pathogens.

\section{References}

1. Baqi M, Mazzulli T. Oligella infections: case report and review of the literature. Can J Infect Dis 1996;7:377-9.

2. Baruah FK, Jain M, Lodha M, Grover RK. Blood stream infection by an emerging pathogen Oligella ureolytica in a cancer patient: case report and review of literature. Indian J Pathol Microbiol 2014;57: 141-3.

3. Cappelli M. Lavaggio dei mitili negli esercizi di vendita. Il Pesce 2010;1:20.

4. Remel. Stuart transport medium. IFU 64620, 2010. Available from: https://tools.thermofisher.com/content/sfs/manuals/IFU64620.pdf

5. Simmons T, Fennelly E, Loughran D. Oligella ureolytica bacteremia in elderly woman. Emerg Infect Dis 2015;21:1271-3.

6. Standard Unit Public Health England. UK standards for microbiology investigations. Identification of Moraxella species and Morphologically similar bacteria. 2015. Available from: http://www. apsi.it/public/ufiles/smi/id13_2_3_rev_en_140311.pdf 